\title{
Saúde e Doença nas Experiências Religiosas/Espirituais: Integrando Modelos de Wilber e Cloninger
}

\author{
Letícia Oliveira Alminhana - Pontifícia Universidade Católica do Rio Grande do Sul, Porto Alegre, Brasil \\ Tiago Pires Tatton-Ramos - Universidade Federal do Rio grande do Sul, Porto Alegre, Brasil \\ Milena Nardini-Bubols - Pontifícia Universidade Católica do Rio Grande do Sul, Porto Alegre, Brasil \\ Luciana Fernandes Marques - Pontifícia Universidade Católica do Rio Grande do Sul, Porto Alegre, Brasil
}

\begin{abstract}
Resumo
O estudo científico das experiências religiosas/espirituais tem sido negligenciado ao longo da história da Psicologia, ocasionando uma indiferenciação destas e dos sintomas psicóticos de conteúdo religioso/espiritual. O objetivo deste artigo foi analisar e comparar duas teorias: a Psicologia Integral, de Wilber, e o Modelo Psicobiológico de Cloninger, a fim de desenvolver critérios para diferenciar saúde e doença mental em experiências religiosas/espirituais. O conceito wilberiano de "Falácia Pré-Trans" aparece enquanto chave de leitura para a diferenciação entre experiência religiosas/espirituais e transtornos mentais, aproximando-se da concepção de maturidade de caráter observada nos conceitos de autodirecionamento e de autotranscendência de Cloninger.

Palavras-chave: experiências religiosas, transpessoal, autodirecionamento, autotranscendência
\end{abstract}

Health and Illness in Religious/Spiritual experiences: integrating Wilber's and Cloninger's Models

\begin{abstract}
The scientific study of religious/spiritual experiences has been neglected throughout the history of psychology causing a lack of differentiation between them and psychotic symptoms. The objective of this study was to analyze and compare two theories: Integral Psychology, by Wilber, and the Psychobiological Model, by Cloninger, in order to distinguish health and mental illness in religious/spiritual experiences. The Wilberian concept of "Pre-Trans Fallacy" is a reading key to differ religious/spiritual experiences from mental disorders. It is similar to the conception of Maturity of Character observed in Cloninger's concepts of Self-directedness and Self-transcendence.

Keywords: Religious experiences; Transpersonal; Self-directedness; Self-transcendence.
\end{abstract}

Salud y Enfermedad en Experiencias Religiosas/Espirituales: integración de los modelos de Wilber y Cloninger

\section{Resumen}

El estudio científico de las experiencias religiosas/espirituales ha sido descuidado a lo largo de la historia de la Psicología causando una indiferencia de éstas y de los síntomas psicóticos de contenido religioso/espiritual. El objetivo de este artículo fue analizar y comparar dos teorías: la Psicología Integral, de Wilber, y el Modelo Psicobiológico de Cloninger, con el fin de desarrollar criterios para diferenciar salud y enfermedad mental en experiencias religiosas/espirituales. El concepto Wilberiano de "Falacia Pre-Trans" aparece como clave de lectura para la diferenciación entre la experiencia religiosa/espiritual y los trastornos mentales, acercándose a la concepción de madurez de carácter observada en los conceptos de Autodirección y Autotrascendencia de Cloninger.

Palabras-clave: Experiencias religiosas; Transpersonal; Autodirección; Autotrascendencia.

Entre as classificações internacionais de transtornos mentais, alguns dos mais graves sintomas encontram-se no âmbito das alucinações e delírios psicóticos (APA, 2013). Ao mesmo tempo, várias experiências psicóticas apresentam, em seu conteúdo, elementos religiosos e/ou espirituais (R/E) (Dalgalarrondo, 2007). Ao longo da história da Psiquiatria e da Psicologia, há um amplo espectro de experiências de caráter $\mathrm{R} / \mathrm{E}$ que foram consideradas como parte de um quadro característico de doença mental (Almeida \& Lotufo Neto, 2004; Moreira-Almeida, Silva de Almeida, \& Neto, 2005). E, embora atualmente se tenha uma compreensão mais abrangente de certos fenômenos, permanece a dificuldade de diferenciação entre experiências $\mathrm{R} / \mathrm{E}$ não patológicas e sintomas psicóticos de conteúdo R/E (Koenig, 2007; Moreira-Almeida \& Cardeña, 2011).

Claridge (2010) afirma que, se as experiências e crenças espirituais são fenomenologicamente semelhantes à visão de mundo psicótica, restam apenas duas posições: 1 - concluir que a religião é apenas uma forma de loucura e que os religiosos são meramente insanos; ou 2 - decidir que as pessoas diagnosticadas como psicóticos podem ser verdadeiramente visionários incompreendidos, rotulados erroneamente e menosprezados por aqueles que não possuem a 
mesma sensibilidade. Segundo o autor, ambas são respostas generalistas. Caso a experiência espiritual e a psicose guardem algumas semelhanças, não significa que tenham a mesma etiologia. É necessário observar criteriosamente o vasto espectro de fatores culturais, sociais, psicológicos e biológicos implicados nessa questão.

William James [1842-1910], em seu livro "Variedades da Experiência Religiosa", define as características da experiência religiosa como: 1) Inefável - tão extraordinária que não pode ser descrita de forma inteligível para nenhuma pessoa que não tenha tido uma experiência semelhante; 2) Noética - a experiência provoca uma forma de insight, trazendo algo como uma "revelação"; 3) Transitória - não dura mais do que meia hora; 4) Passiva - a experiência não pode ser "criada" e as pessoas relatam terem sido "tomadas por algo" (James, 1929).

Em virtude das semelhanças fenomenológicas entre sintomas psicóticos e algumas experiências $\mathrm{R} / \mathrm{E}$ não patológicas, Grof e Grof $(1989,1997)$ criaram o conceito de emergências espirituais (spiritual emergences) a fim de nomear crises existenciais em que experiências $\mathrm{R} / \mathrm{E}$ poderiam emergir tanto de uma forma saudável (spiritual emergence) quanto perturbadora (spiritual emergency). Outros autores também têm apontado uma série de terminologias utilizadas para identificar experiências que se assemelham a sintomas psicóticos, embora não sejam necessariamente patológicas (Cardeña, Lynn, \& Krippner, 2004). Mais recentemente, o termo "experiência anômala" tem sido proposto para nomear - sem assumir implicações psicopatológicas - vivências incomuns, diferentes do habitual ou das explicações usualmente aceitas como realidade (Cardeña et al., 2004; Martins \& Zangari, 2012).

Alguns estudos foram conduzidos no Brasil visando compreender as experiências anômalas em contexto religioso, trazendo importantes contribuições a esse tema (Menezes Jr., Alminhana, \& Moreira-Almeida, 2012; Moreira-Almeida, Lotufo Neto, \& Greyson, 2007). Em uma revisão sistemática, Menezes Jr e Moreira-Almeida apresentam "Nove Critérios Diferenciais entre Experiências Religiosas e Transtornos Mentais". Embora seja um importante esforço em direção a maior compreensão do fenômeno, os Nove Critérios não foram respaldados em evidências empíricas e, mesmo após terem sido testados, apenas a metade mostrou correlação com saúde mental, carecendo de mais estudos (Menezes Jr, 2012; Menezes Jr \& Moreira-Almeida, 2009).
Frente a esse cenário, o principal objetivo deste artigo é apresentar duas teorias de personalidade que integram a espiritualidade em seu modelo. Estas sugerem critérios teóricos e experimentais que permitem uma compreensão mais ampla das diferenças entre saúde e doença mental nas experiências anômalas de caráter R/E (Cloninger, Svrakic \& Przybeck, 1993; Wilber, 2000b).

\section{A Abordagem Integral em Psicologia: Ken Wilber e a "Falácia Pré-Trans"}

A chamada Abordagem Integral reflete uma tentativa teórica de sistematização capaz de integrar, de maneira harmoniosa, diferentes perspectivas psicológicas sobre a consciência (Tatton-Ramos \& Alminhana, 2007; Wilber, 1996, 2000a). A partir de uma volumosa revisão de literatura sobre diferentes estados de consciência, Ken Wilber desenvolveu um modelo teórico que denominou de "Abordagem Integral em Psicologia". Pautado em uma fenomenologia similar àquela de Eliade (1959), ou seja, por meio da busca de elementos essenciais em diferentes tradições místicas - do ocidente e do oriente - a Abordagem Integral promoveu um embate inovador entre Psicologia, R/E e saúde mental (Wilber, 2000a). Embora vários livros do autor tenham sido traduzidos para o português, suas discussões epistemológicas ainda não tiveram inserção importante no âmbito acadêmico.

No trabalho de Wilber, Engler, \& Brown (1991), realizado com o apoio de psiquiatras de Harvard como Jack Engler e Daniel P. Brown, é elaborada uma lista de possíveis psicopatologias para diferentes níveis de desenvolvimento do ego (da noção de "eu"). Estes incluem desde processos psicológicos básicos até experiências R/E ou anômalas. Portanto, ao invés de ignorar essa suposta dimensão da experiência religiosa, os autores tomam como fenomenologias válidas as experiências de místicos ocidentais e orientais, tais como São João da Cruz, Eckhart, Sri Aurobindo e Nagarjuna. Assim procedendo, retomam e ampliam o caminho trilhado décadas antes por um dos pais da Psicologia, William James, no clássico "As variedades da Experiência Religiosa" (James, 1929). Também contribuem com a chamada Psicologia da Religião, da década de 1880, a qual sempre esteve presente na história da Psicologia (Paiva, 2002).

Conceitos ainda recentes - porém pouco conhecidos - desenvolvidos pela Abordagem Integral, como "falácia pré-trans" e "ciência ampla", tratam da dificuldade em legitimar o grau de fidedignidade de uma 
experiência R/E (Wilber, 2000b). Essa "fidedignidade" seria um reflexo da organização da experiência $\mathrm{R} / \mathrm{E}$ ao nível do ego em que é vivenciada (Wilber et al., 1991). Por exemplo, uma experiência que pode ser interpretada como "falar com Deus", dependendo do nível de organização egoica do indivíduo que a experimenta, pode ser tanto a consequência de um adoecimento psíquico quanto uma vivência mística ou transcendente potencialmente saudável (Wilber et al., 1991). Essa compreensão, somada à instrumentalização de epistemologias teológicas, como a teoria dos "três olhos do conhecimento" de São Boaventura, levada a cabo pela Abordagem Integral, permite o desenvolvimento de ferramentas que proporcionam um avanço para as pesquisas sobre as relações entre experiências $\mathrm{R} / \mathrm{E}$ e saúde (Wilber, 2000b, 2001; Wilber et al., 1991).

\section{Falácia Pré-Trans}

Segundo Wilber (1996, 2000a), a personalidade se desenvolve por meio de diferentes estruturas e níveis, que vão desde camadas pré-egoicas até a dimensão transegoica, passando - propriamente - pela formação do eu (nível egoico). Manifestações comuns ao nível transegoico podem incluir experiências anômalas de diferentes ordens, incluindo aquelas observadas na esfera da $\mathrm{R} / \mathrm{E}$, exemplarmente representadas pela vivência de êxtase místico (Walsh \& Vaughan, 1994; Wilber et al., 1991). Ao incluir a investigação desse nível no desenvolvimento da personalidade, Wilber se coloca ao lado de uma longa e respeitada tradição da Psicologia que inclui estudiosos, como William James, Erik Erikson, Erich Fromm, Carl G. Jung, Viktor Frankl, A. Maslow e M. Seligman (Argyle, 1999; Paloutzian \& Park, 2013; Wulff, 1997). Essa perspectiva contrapõese a modelos que - peremptoriamente - compreendem as experiências $\mathrm{R} / \mathrm{E}$ como formas gerais de neuroses, ilusões, distorções cognitivas e infantilidade do pensamento, como a interpretação freudiana clássica (Freud, 2001) e os demais reducionismos (Dawkins, 2007; Dennett, 2007).

Em seu modelo, o nível pré-egoico resume as teorias clássicas do desenvolvimento, compreendendo o pré-ego como o período que abrange as vivências básicas da infância. Parte dessas vivências, na criança, envolve não apenas as dificuldades de diferenciação "eu-outro" e formação de uma identidade, mas também a imersão em um universo de fantasias, tais como experiências que podem incluir o diálogo com "amigos invisíveis" e universos autorreferenciais. $\mathrm{Na}$ criança, ou seja, no pré-ego, tais experiências são naturais e quase sempre saudáveis, sendo parte natural do processo de formação do eu. Porém, no adulto, vivências pré-egoicas estão associadas a experiências psicotizantes, que podem incluir alucinações e delírios de conteúdo R/E ou não (p. ex., delírios de onipotência onde o indivíduo acredita que está em contato direto com Deus).

A novidade no modelo de Wilber (2008) está na investigação das estruturas trans-egoicas que transcendem a formação de um eu saudável. As vivências do tipo trans-egoicas estão associadas ao bem-estar, qualidade de vida e estruturação saudável da personalidade. Podem incluir estados místicos, de bem-aventurança e diversas vivências R/E (Wilber, 2008). A principal diferença entre uma experiência de caráter pré-egoico e outra de caráter transegoico é a presença ou não de um ego saudável capaz de traduzi-la de forma coerente, madura, atribuindo-lhe um sentido que signifique e integre essa experiência na vida do indivíduo como um todo.

Um trecho de Campbell (1995), resume de forma poética as ideias apresentadas acima:

O mistico, dotado de talentos inatos e seguindo a instrução de um mestre, entra na água e descobre que sabe nadar; o esquizofrênico, por sua vez, despreparado, sem orientação e sem dotes, caiu nela, ou mergulbou voluntariamente, e está se afogando (Campbell, 1995, p.119).

Wilber (1996; 2008), no entanto, aponta para um problema central no entendimento tradicional da Psicologia em relação ao amplo espectro de experiências $\mathrm{R} / \mathrm{E}$ - a chamada falácia pré-trans. O autor chama a atenção para o fato de que a influência das teorias psicodinâmicas induziu a uma classificação incorreta das experiências $\mathrm{R} / \mathrm{E}$ na perspectiva da saúde, já que estas tendem a confundir estruturas de desenvolvimento pré-egoicas com as transegoicas (Wilber, 1996). Isso ocorreria quando uma experiência de caráter pré-egoica é erroneamente classificada como uma experiência transegoica, e vice-versa, justamente pela semelhança fenomenológica das vivências descritas em cada uma delas (Wilber, 1996).

Talvez devido à sua eloquência metafísica, linguagem um tanto esotérica e, especialmente falta de pesquisa experimental, a Abordagem Integral ainda não está devidamente inserida no meio acadêmico. Permanece, portanto, como uma engenhosidade filosófica e teórica que, como pensador pode auxiliar na reflexão sobre as relações entre personalidade e espiritualidade. Não obstante, o modelo é parcialmente resgatado por C. Robert Cloninger para o desenvolvimento do 
modelo Psicobiológico do Temperamento e Caráter (Cloninger et al., 1993).

\section{Robert Cloninger e o Modelo Psicobiológico de Temperamento $e$ Caráter}

O Modelo Psicobiológico é dividido em dois aspectos fundamentais: o Temperamento e o Caráter (Cloninger, Bayon \& Svrakic, 1998; Cloninger et a., 1993). De acordo com Cloninger, o Temperamento reúne as bases neurogenéticas que fundamentam a personalidade e pode ser observado por meio de respostas automáticas em relação a estímulos emocionais. As características de temperamento de cada indivíduo determinarão as diferenças em suas respostas emocionais habituais, como medo, raiva e aversão. As dimensões do temperamento identificadas por Cloninger (2004) são: Busca por Novidade; Evitação por Danos; Dependência de Recompensa e Persistência.

Observando que características como maturidade, flexibilidade e intuição não eram contempladas em seu modelo de personalidade, Cloninger (2004) comparou a dinâmica do temperamento com sistemas que descreviam o caráter humano, como a Psiquiatria Psicodinâmica e as Psicologias Humanistas e Transpessoal. Depois disso, concluiu que o "Caráter" seria o reflexo de objetivos e valores pessoais, surgindo por meio das interações do indivíduo com a realidade que o cerca (relações sujeito-objeto).

Dessa forma, o caráter seria a consequência de três aspectos do desenvolvimento de autoconceitos: 1) o quanto uma pessoa é capaz de se ver como um indivíduo autônomo; 2) como uma parte integral da humanidade ou da sociedade; 3) como uma parte integral do Universo, como um "todo". Assim sendo, cada aspecto de autoconceito, corresponde a uma das três dimensões de Caráter: 1) Autodirecionamento; 2) Cooperatividade; 3) Autotranscendência (Cloninger et al., 1993).

- Autodirecionamento: quando um indivíduo é capaz de controlar, regular e adaptar o comportamento para se ajustar à determinada situação, de acordo com objetivos e valores que ele mesmo tenha escolhido. Capacidade de autocrítica, sentido de propósito na vida, capacidade de adiar gratificações e superação de desafios (Cloninger et al., 1993). Baixo autodirecionamento é a característica comum entre os transtornos de personalidade, os transtornos psicóticos e de humor (Cloninger et al., 1993; Gonzalez-Torres et al., 2009;
Gutiérrez-Zotes, Córtez, Valero, Peña, \& Labad, 2005).

- Cooperatividade: capacidade de identificar-se e de aceitar as outras pessoas; sociabilidade que é oposta ao egocentrismo e à hostilidade. Pessoas cooperativas são descritas como tolerantes, empáticas, solícitas e compassivas (Cloninger et al., 1993).

- Autotranscendência (self-aware consciousness): Fundamentada na Psicologia Humanista, Transpessoal e Integral (Cloninger et al., 1993). Apesar da importância dessas experiências, elas têm sido sistematicamente negligenciadas e omitidas de inventários de personalidade (Alminhana \& Moreira-Almeida, 2009). Autotranscendência se refere à capacidade de "esquecer" de si mesmo, indo além dos impulsos egoístas e familiares. Possibilidade de aceitar a existência de relações que podem estar além das soluções meramente analíticas e dedutivas, como ocorre em experiências relacionadas à espiritualidade (Cloninger et al., 1993).

Assim definido, o Caráter envolveria a aprendizagem baseada em insight ou conceitual que emerge após a aprendizagem pré-conceitual e está associada ao temperamento (Cloninger, 1993). Segundo Cloninger, Svrakic e Przybeck (1993), o desenvolvimento da personalidade é um processo epigenético interativo onde os fatores hereditários do temperamento motivam a aprendizagem por insight, a qual, por sua vez, modifica a significância do estímulo percebido.

A partir disso, Cloninger et al. (1993), desenvolveram o Inventário de Temperamento e Caráter (ITC), a fim de mensurar quantitativamente as sete dimensões de personalidade. O ITC tem sido administrado em inúmeros estudos, em diversos países e, por integrar a espiritualidade como um constructo ligado à maturidade do caráter, tem apresentado resultados importantes para o avanço da compreensão das relações entre personalidade e espiritualidade (Cloninger et al., 1993; Cloninger, Svrakic, \& Svrakic, 1997; Guillem, Bicu, Semkovska, \& Debruille, 2002; Hori et al., 2008).

\section{Autotranscendência: Sanidade ou Loucura?}

A Autotranscendência (AT) é a dimensão do caráter que mais tem apresentado resultados controversos em pesquisas que investigam as relações entre personalidade e transtornos mentais. Vários estudos 
sobre esquizofrenia e esquizotipia têm apresentado um mesmo perfil de personalidade relacionado à presença de transtornos mentais: baixos escores em autodirecionamento (AD), baixos escores em cooperatividade (C) e altos escores em autotranscendência (AT) (Bayon, Hill, Svrakic, Przybeck, \& Cloninger, 1996; Bora \& Veznedaroglu, 2007; Daneluzzo, Stratta, \& Rossi, 2005; Gonzalez-Torres et al., 2009; Hori et al., 2008). Hori et al. (2008) encontraram associações entre esquizofrenia, baixos escores em AD e altos escores em AT, sugerindo que esta última seria uma dimensão da personalidade substancialmente relacionada com a gravidade dos sintomas positivos da doença.

Tais resultados parecem se contrapor à teoria desenvolvida no Modelo Psicobiológico (Cloninger et al., 1993). Segundo esse modelo, as três dimensões do caráter (AD, C e AT) estariam apontando em direção à maturidade. A dimensão de autotranscendência (AT) foi especificamente relacionada às capacidades de ajustamento, satisfação pessoal e ir além do autocentramento e do pensamento analítico (Cloninger et al., 1993). Por tudo isso, observa-se uma aparente contradição entre a proposta de maturidade do "eu" em relação à natureza e a totalidade do universo (altos escores em AT) e os achados que mostram alta AT como indicativo de presença de transtornos mentais ou de tendência para desenvolvê-los. Tal contradição reforça a dificuldade de compreender e diferenciar experiências de caráter $\mathrm{R} / \mathrm{E}$ saudáveis de sintomas psicopatológicos, também apresentada na falácia pré-trans de Wilber (2008).

Ao mesmo tempo, Guillem, Bicu, Semkovska e Debruille (2002) afirmam que altos escores em AT parecem caracterizar indivíduos que são capazes de tolerar a ambiguidade e que devem experimentar pensamento mágico e associações em consonância com os sintomas psicóticos. Em outro estudo, os autores sugerem que AT pode estar associada a dois constructos diferentes: um ligado à propensão à psicose, com imaturidade de caráter; e outro relacionado à "criatividade madura", ou seja, abertura para sentimentos, pensamentos e comportamentos incomuns e também facilidade para oferecer significados incomuns e conexões imaginativas para as experiências. Assim, o que irá definir adoecimento ou saúde mental será a relação de AT com as demais características, principalmente aquelas ligadas ao desenvolvimento de maturidade do caráter (altos escores em AD e C) (Daneluzzo et al., 2005).

Pessoas com alta AT que conseguiram desenvolver também uma estrutura de "eu" ou de "ego", autonomia, responsabilidade, capacidade de relacionar-se com os demais (altos $\mathrm{AD}, \mathrm{C}$ e AT), apresentam melhores níveis de saúde mental, criatividade e espiritualidade (Cloninger \& et al., 1993; Cloninger, 2004; Fodor, 1995). Por outro lado, indivíduos que apresentam alta AT, mas não conseguiram estruturar-se como indivíduos autônomos, possuem a tendência de culpar os outros ao invés de assumir responsabilidade por seus comportamentos e apresentam dificuldades para desenvolver relações interpessoais maduras e duradouras (baixos AD e C, embora alta AT), apresentam forte tendência para o desenvolvimento de uma série de transtornos mentais (Cloninger et al., 1993; Bayon et al., 1996; Cloninger, 2004).

As relações entre AT e transtornos mentais já eram apresentadas de forma clara por Cloninger e seus colegas desde seus primeiros estudos (Cloninger et al., 1993; Bayon et al., 1996). Nestes, os autores mencionam que AT seria particularmente útil para diagnosticar pacientes esquizotípicos, já que estes possuem a tendência de sustentar questões sobre percepção extrasensorial e demais elementos que apresentam fenomenologia similar às experiências anômalas observadas em certas manifestações de R/E (Cloninger et al., 1993).

Além disso, nesses estudos, os autores analisaram as relações entre os escores do ITC e os transtornos clínicos e de personalidade (Eixo I e II do DSM-III). Os resultados confirmaram que baixos escores em $\mathrm{AD}$ e C parecem ser as características essenciais associadas a todos os transtornos de personalidade. Além disso, alta AT está associada à tendência a ver significados não usuais e conexões imaginativas nas experiências vividas (Bayon et al., 1996).

Em resumo, tudo indica que o conceito de autotranscendência (AT) constitui uma medida confiável para a compreensão das experiências relacionadas a $\mathrm{R} / \mathrm{E}$. Igualmente, quando AT é observada isoladamente, é difícil classificá-la como um indicador de maturidade (saúde) ou imaturidade (patologia). A fenomenologia presente na experiência do místico, por exemplo, se analisada isoladamente, é pouco elucidativa, posto suas semelhanças com a experiência da loucura. Apenas a associação dessas vivências com os níveis de autodirecionamento (AD) e de cooperatividade $(\mathrm{C})$ é que poderão apontar de maneira acurada para as consequências de adoecimento ou saúde (Bayon et.al., 1996). Mais especificamente: altos escores em AT, $\mathrm{AD}$ e $\mathrm{C}$ sugerem saúde; altos escores em AT e baixos escores em $\mathrm{AD}$ e $\mathrm{C}$ sugerem adoecimento (Cloninger et al., 1993; Bayon et al., 1996). 
Os Construtos de "Falácia Pré-trans", "Autodirecionamento" e "Autotranscendência": Visões Complementares sobre Saúde Mental e $\mathrm{R} / \mathrm{E}$

Os dois modelos de personalidade apresentados anteriormente, a Abordagem Integral e o Modelo Psicobiológico de Temperamento e Caráter, mostram pontos de encontro fundamentais para o entendimento das relações entre experiências $\mathrm{R} / \mathrm{E}$ e níveis de saúde/adoecimento. Apesar de Wilber e Cloninger adotarem perspectivas epistemológicas distintas, os modelos possuem pontos de contato e, em grande parte, as duas teorias apresentam semelhanças. Conceitos semelhantes, tais como: maturidade do ego ("nível egoico", em Wilber, e "autodirecionamento", em Cloninger) e vivência de experiências $\mathrm{R} / \mathrm{E}$ (vivências transegoicas, em Wilber, e experiências de autotranscendência, em Cloninger).

Como apresentado anteriormente, Cloninger (2004) chama a atenção para a correspondência entre o que ele define como "desenvolvimento da dimensão do caráter" e o que é chamado - em outras teorias de maturação cognitiva, desenvolvimento psicossexual ou desenvolvimento do ego. Tais relações recebem suporte de estudos que mostram que os traços do caráter estão, de fato, fortemente relacionados à maturidade das defesas do ego (Mulder, Joyce, Sellman, Sullivan, \& Cloninger, 1996). Nesse sentido, Holt et al. (2008) e Claridge (2010) apontam para características chamadas de "força do ego", ou uma tendência a não ver as coisas de forma autorreferenciada. Investigando a influência da propensão à psicose e da força do ego na criatividade de estudantes universitários, Fodor (1995), define força do ego como a capacidade de tolerar e de se realizar por meio da independência e flexibilidade.

Em se tratando de força do ego (Fodor), Autodirecionamento (Cloninger) ou Ego maduro (Wilber), observa-se a importância central da estruturação da noção de "eu" (ego), sendo este o elemento central que direciona as experiências para a saúde ou para a doença. Dessa forma, altos escores em Autotranscendência isoladamente - podem sugerir características do nível pré-egoico de Wilber, associadas ao espectro das psicoses (Wilber et al., 1991). Essa relação é particularmente forte quando essas vivências não estão associadas com maturidade do "eu" (altos escores em Autodirecionamento (AD) no modelo de Cloninger). Por outro lado, quando experiências de AT estão associadas à maturidade de caráter (altos escores em AD e C) é provável uma relação com a saúde e o bem-estar do nível transegoico descrito por Wilber (Wilber, 2000a).
A confusão ou falácia poderá ocorrer quando se observa unicamente a relação entre alta AT e experiências psicóticas, desconsiderando-se o papel fundamental da força do ego ou, especificamente, do Autodirecionamento nas experiências anômalas ou R/E (Bayon et al., 1996; Bora \& Veznedaroglu, 2007; Daneluzzo et al., 2005; Gonzalez-Torres et al., 2009; Smith, Riley, \& Peters, 2009; Svrakic et al., 2002; Hori et al., 2008).

\section{Conclusão}

Este artigo versou sobre as relações entre quatro temas centrais: 1 - o modelo da Abordagem Integral, de Ken Wilber; 2 - o Modelo Psicobiológico do Temperamento e Caráter, de Cloninger; 3 - experiências anômalas e R/E; 4 - os conceitos de saúde e adoecimento. Buscou-se oferecer critérios teóricos e experimentais mais robustos e atuais para avaliação das relações entre experiências $\mathrm{R} / \mathrm{E}$ e desfechos de saúde. Foi possível perceber nos dois modelos não apenas a inclusão de elementos $\mathrm{R} / \mathrm{E}$ como fatores importantes no desenvolvimento da personalidade, mas também as propostas similares e complementares no que tange a diferenciação entre experiências do tipo saudáveis e patológicas (Wilber \& colaboradores, 1991; Wilber, 2008; Cloninger et al., 1993).

Assim sendo, talvez seja possível responder às indagações levantadas por Claridge (2010), quando traz à tona a questão sobre o que diferencia duas pessoas que possuem a mesma propensão a experiências anômalas, sendo uma levada ao transtorno e outra à "inspiração", espiritualidade ou criatividade. Quais seriam os determinantes para isso?

Em resposta à indagação, é legítimo pensar que os parâmetros oferecidos pela análise da estrutura da personalidade possam servir como uma ferramenta importante para diferenciar um quadro de psicopatologia de uma experiência $\mathrm{R} / \mathrm{E}$ saudável. Os estudos têm evidenciado o papel do desenvolvimento do caráter, enquanto maturidade do "eu" (AD); do "nós" (C) e do "todo" (AT), na diferenciação entre saúde e doença mental (Bayon et al., 1996; Bora \& Veznedaroglu, 2007; Daneluzzo et al., 2005; Gonzalez-Torres et al., 2009; Svrakic et al., 2002; Hori et al., 2008).

Conclui-se assim, que a mera observação e descrição de uma determinada experiência religiosa/ espiritual, ou mesmo a observação dos critérios sintomatológicos apresentados em manuais de transtornos mentais, como o DSM (American Psychiatric Association, 2013), parecem limitados para classificar 
uma experiência desse tipo como saudável ou patológica (Moreira-Almeida \& Cardeña, 2011). Quando um indivíduo relata experiências anômalas de conteúdo R/E (autotranscendência), é preciso examinar a maturidade de ego, o nível de autodirecionamento, a capacidade de independência, responsabilidade e autocrítica. Também é preciso avaliar o nível de cooperatividade, de tolerância e aceitação das diferenças, de empatia e capacidade de relacionamento social. Ao que tudo indica, somente agregando esses critérios, pode-se avaliar com mais clareza a natureza saudável (transegoica) ou patológica (pré-egoica) da experiência. Futuros estudos poderão verificar a viabilidade de tais apontamentos por meio de análises teórico-experimentais, especialmente utilizando as ferramentas aqui apresentadas, a saber: os conceitos teóricos da Abordagem Integral e o Inventário de Temperamento e Caráter (ITC). Desse modo, será possível reunir elementos teóricos fundamentais com um instrumento capaz de mensurar e avaliar empiricamente as relações entre experiências $\mathrm{R} / \mathrm{E}$ e transtornos mentais.

\section{Referências}

Almeida, A. M. de, \& Lotufo Neto, F. (2004). Mediumship seen by some pioneers of mental health. Revista de Psiquiatria Clínica, 31(3), 132-141. doi: 10.1590/S0101-60832004000300003

Alminhana, L. O., \& Moreira-Almeida, A. (2009). Personality and religiousness/spirituality (R/E). Revista de Psiquiatria Clínica, 36(4), 153-161. doi: 10.1590/S0101-60832009000400005

American Psychiatric Association. (2013). Diagnostic and statistical manual of mental disorders, 5th Edition: DSM-5 (5 edition). Washington, D.C: American Psychiatric Publishing.

Argyle, M. (1999). Psychology and religion: An introduction. London; New York: Routledge.

Bayon, C., Hill, K., Svrakic, D. M., Przybeck, T. R., \& Cloninger, C. R. (1996). Dimensional assessment of personality in an out-patient sample: Relations of the systems of Millon and Cloninger. Journal of Psychiatric Research, 30(5), 341-352. Recuperado de http:/ /www.ncbi.nlm.nih.gov/pubmed/8923338 http://dx.doi.org/10.1016/0022-3956(96)00024-6

Bora, E., \& Veznedaroglu, B. (2007). Temperament and character dimensions of the relatives of schizophrenia patients and controls: The relationship between schizotypal features and personality. European Psychiatry: The Journal of the Association of European Psychiatrists, 22(1), 27-31. doi: 10.1016/j. eurpsy.2006.07.002

Campbell, J. (1995). Myths to live by. London: Souvenir Press Ltd.

Cardeña, E., Lynn, S. J., \& Krippner, S. (2004). Varieties of anomalous experience: Examining the scientific evidence. Washington, DC: American Psychological Association.

Claridge, G. (2010). Spiritual experience: Healthy psychoticism? Em Clarke (Ed.), Psychosis and Spirituality (pp. 75-87). John Wiley \& Sons, Ltd. Recuperado de http://onlinelibrary.wiley.com/ doi/10.1002/9780470970300.ch7/summary

Cloninger, C. R. (2004). Feeling good: The science of wellbeing. Oxford; New York: OUP USA.

Cloninger, C. R., Bayon, C., \& Svrakic, D. M. (1998). Measurement of temperament and character in mood disorders: A model of fundamental states as personality types. Journal of Affective Disorders, 51(1), 21-32. doi: 10.1016/S0165-0327(98)00153-0

Cloninger, C. R., Svrakic, D. M., \& Przybeck, T. R. (1993). A psychobiological model of temperament and character. Archives of General Psychiatry, 50(12), 975990. doi: 10.1001/archpsyc.1993.01820240059008

Cloninger, C. R., Svrakic, N. M., \& Svrakic, D. M. (1997). Role of personality self-organization in development of mental order and disorder. Development and Psychopathology, 9(04), 881-906. Recuperado de http://www.ncbi.nlm.nih.gov/pubmed/9449010

Dalgalarrondo, P. (2007). Brazilian studies on religion and mental health: History and current perspectives. Revista de Psiquiatria Clínica, 34, 25-33. doi: 10.1590/S0101-60832007000700005

Daneluzzo, E., Stratta, P., \& Rossi, A. (2005). The contribution of temperament and character to schizotypy multidimensionality. Comprehensive Psychiatry, 46(1), 50-55. doi: 10.1016/j.comppsych.2004.07.010

Dawkins, R. (2007). The God Delusion (New Ed with additions edition). London: Black Swan.

Dennett, D. C. (2007). Breaking the spell: Religion as a naturalphenomenon (Reprint edition). London: Penguin.

Eliade, M. (1959). The sacred and the profane: The nature of religion. New York: Harcourt Australia. 
Fodor, E. M. (1995). Subclinical manifestations of psychosis-proneness, ego strength, and creativity. Personality and Individual Differences, 18(5), 635-642. doi: 10.1016/0191-8869(94)00196-Y

Freud, S. (2001). Complete psychological works of sigmund freud, The Vol 21: "The Future of an Illusion", "Civilization and Its Discontents" and Other Works Vol 21 (New Ed edition). London: Vintage Classics.

Gonzalez-Torres, M. A., Inchausti, L., Ibáñez, B., Aristegui, M., Fernández-Rivas, A., Ruiz, E., ... Bayón, C. (2009). Temperament and character dimensions in patients with schizophrenia, relatives, and controls: The Journal of Nervous and Mental Disease, 197(7), 514-519. doi: 10.1097/NMD.0b013e3181aacc1a

Grof, S., \& Grof, C. (1989). Spiritual emergency: When personal transformation becomes a crisis. Los Angeles: New York: Warner Books.

Grof, C., \& Grof, S. (1997). Stormy search for the self, the: A guide to personal growth through transformational crisis (Reprint edition). Flushing, N.Y.: Jeremy P Tarcher.

Guillem, F., Bicu, M., Semkovska, M., \& Debruille, J. B. (2002). The dimensional symptom structure of schizophrenia and its association with temperament and character. Schizophrenia Research, 56(1-2), 137-147. doi: 10.1016/S0920-9964(01)00257-2

Gutiérrez-Zotes JA, Cortés MJ, Valero J, Peña J, \& Labad A. (2005). Psychometric properties of the abbreviated Spanish version of TCI-R (TCI-140) and its relationship with the Psychopathological Personality Scales (MMPI-2 PSY-5) in patients. Actas Esp Psiquiatr. 33(4), 231-237.

Holt, N., Simmonds-Moore, C., Moore, S., Holt, N., Simmonds-Moore, C., \& Moore, S. (2008). Benign schizotypy: Investigating differences between clusters of schizotype on paranormal belief, creativity, intelligence and mental health. Em S. Sherwood \& S. Sherwood (Eds.), Proceedings of Presented Papers: The Parapsychological Association 51st Annual Convention (pp. 82-96). Parapsycholgical Association. Recuperado de http:/ / eprints.uwe.ac.uk/15726/

Hori, H., Noguchi, H., Hashimoto, R., Nakabayashi, T., Saitoh, O., Murray, R. M., ... Kunugi, H. (2008). Personality in schizophrenia assessed with the Temperament and Character Inventory (TCI). Psychiatry Research, 160(2), 175-183. doi: 10.1016/j. psychres.2007.05.015
James, W. (1929). The varieties of religious experience. London: Modern Library.

Koenig, H. G. (2007). Religião, espiritualidade e psiquiatria: Uma nova era na atenção à saúde mental. Revista de psiquiatria clínica. (São Paulo), 34(supl.1), 5-7. doi: 10.1590/S0101-60832007000700002

Martins, L. B., \& Zangari, W. (2012). Relations among typical contemporary anomalous experiences, mental disorders and spiritual experiences. Revista de Psiquiatria Clínica, 39(6), 198-202. doi: 10.1590/ S0101-60832012000600004

Menezes Jr., A. (2012). Estudo prospectivo sobre o diagnóstico diferencial entre experiências mediunicas e transtornos mentais de conteúdo religioso (Tese de doutorado em Saúde). Faculdade de Medicina, Universidade Federal de Juiz de Fora, Juiz de Fora/MG.

Menezes Jr., A., Alminhana, L., \& Moreira-Almeida, A. (2012). Perfil sociodemográfico e de experiências anômalas em indivíduos com vivências psicóticas e dissociativas em grupos religiosos. Revista de Psiquiatria Clínica, 39(6), 203-207. doi: 10.1590/ S0101-60832012000600005

Menezes Jr., A., \& Moreira-Almeida, A. (2009). Differential diagnosis between spiritual experiences and mental disorders of religious content. Archives of Clinical Psychiatry (São Paulo), 36(2), 75-82. doi: 10.1590/S0101-60832009000200006

Moreira-Almeida, A., \& Cardeña, E. (2011). Differential diagnosis between non-pathological psychotic and spiritual experiences and mental disorders: A contribution from Latin American studies to the ICD-11. Revista Brasileira de Psiquiatria, 33, s21-s28. doi: 10.1590/S1516-44462011000500004

Moreira-Almeida, A., Lotufo Neto, F., \& Greyson, B. (2007). Dissociative and psychotic experiences in Brazilian spiritist mediums. Psychotherapy and Psychosomatics, 76(1), 57-58. doi: 10.1159/000096365

Mulder, R. T., Joyce, P. R., Sellman, J. D., Sullivan, P. F., \& Cloninger, C. R. (1996). Towards an understanding of defense style in terms of temperament and character. Acta Psychiatrica Scandinavica, 93(2), 99-104. doi: 10.1111/j.1600-0447.1996.tb09809.x

Paiva, G. J. Ciência, religião, psicologia: Conhecimento e comportamento. Psicologia: Reflexão e Crítica, 15(3), 561-567. 
Paloutzian, R. F., \& Park, C. L. (2013). Handbook of the psychology of religion and spirituality, second edition (Second Edition edition). New York, NY: The Guilford Press.

Smith, L., Riley, S., \& Peters, E. R. (2009). Schizotypy, delusional ideation and well-being in an American new religious movement population. Clinical Psychology \& Psychotherapy, 16(6), 479-484. doi: 10.1002/ cpp. 645

Svrakic, D. M., Draganic, S., Hill, K., Bayon, C., Przybeck, T. R., \& Cloninger, C. R. (2002). Temperament, character, and personality disorders: etiologic, diagnostic, treatment issues. Acta Psychiatrica Scandinavica, 106(3), 189-195. doi: 10.1034/j.1600-0447.2002.02196.x

Tatton-Ramos, T., \& Alminhana, L. O. (2007). Por uma epistemologia maior - $\mathrm{O}$ "essencialismo" como necessidade na sociologia transcendental de Ken Wilber. Revista Virtú-ICH. 5 ed. ISSN 18089011. Recuperado de http://www.ufff.br/virtu/ files/2010/05/5a-1.pdf

Walsh, R. N., \& Vaughan, F. (1994). Paths beyond ego: Transpersonal vision. Los Angeles, CA: Warner Books.
Wilber, K. (1996). The spectrum of consciousness (2nd edition edition). Wheaton, IL USA: Quest Books, U.S.

Wilber, K. (2000a). Integral psychology: Consciousness, spirit, psychology, therapy (1st Pbk. Ed edition). Boston: Shambhala Publications Inc.

Wilber, K. (2000b). The marriage of sense and soul: Integrating science and religion. New York: Broadway Books.

Wilber, K. (2001). Eye to eye: The quest for the new paradigm (New edition edition). Boston: New York: Shambhala Publications Inc.

Wilber, K. (2008). Collected Works of Ken Wilber: v.2: Atman Project, Up from Eden, Selected Essays: Vol 2. Boston: Shambhala Publications Inc.

Wilber, K., ..., Engler, J., \& Brown, D. (1991). Transformations of consciousness: Conventional and contemplative perspectives on development (1 edition). Boston: New York: Shambhala Publications Inc.

Wulff, D. H. (1997). Psychology of religion: Classic and contemporary views (2nd Edition). New York: John Wiley \& Sons.

Recebido em: 22/09/2015 Reformulado em: 15/02/2016; 28/03/2016

Aceito em: $31 / 03 / 2016$ 
Sobre os autores:

Letícia Oliveira Alminhana é pós-doutora pela University of Oxford/UK, doutora em Saúde pela UFJF/MG, mestre em Teologia pela EST/RS e psicóloga pela PUCRS. Atualmente é bolsista PNPD/CAPES na pós-graduação da Psicologia da PUCRS, coordenadora do grupo de estudos e pesquisas em Saúde, Espiritualidade e Religiosidade (GepSER - PUCRS), e membro do Núcleo de Psiquiatria e Espiritualidade (NUPE/APRS) e do GT Psicologia e Religião da ANPPEP.

E-mail: leticia.alminhana@pucrs.br ou leticiaalminhana@gmail.com.

Tiago Pires Tatton-Ramos é psicólogo, com especialização e mestrado em Ciência da Religião, pela Universidade Federal de Juiz de Fora/MG, doutorando em Psicologia, pela Universidade Federal do Rio Grande do Sul e membro do Laboratório de Fenomenologia Experimental e Cognição (LAFEC - UFRGS), com Estágio de Doutorado no King's College/UK.

E-mail: tiagotatton@gmail.com

Milena Nardini-Bubols é graduada em Psicologia pela PUCRS, especialista em Psicologia Transpessoal (UNIPAZ/SUL) e mestre em Psicologia Social e da Personalidade (PUCRS). É coordenadora do Comitê de Psicologia Transpessoal da Sociedade de Psicologia do Rio Grande do Sul (SPRGS), colaboradora do GepSER e do Núcleo de Psiquiatria e Espiritualidade (NUPE/APRS) e atua como psicóloga clínica em consultório particular, com ênfase em Psicologia Transpessoal.

Luciana Fernandes Marques é psicóloga pela PUCRS, mestre em Psicologia Social e da Personalidade pela PUCRS, doutora em Psicologia pelo PUCRS, com pós-doutorado em Psicologia pela UFRGS e em Psicologia ISCTE-IUL, Lisboa. É professora adjunta IV da Faculdade de Educação da UFRGS, professora permanente do Programa de PósGraduação Ensino na Saúde (Faculdade de Medicina UFRGS) e membro do GT Psicologia e Religião da ANPPEP. E-mail: luciana.marques@ufrgs.br

Contato com os autores:

Letícia Oliveira Alminhana

Rua Vicente da Fontoura, 1190 apartamento 401 - Bairro Rio Branco

Porto Alegre-RS, Brasil

CEP: 90640-000

Cel.: (51) 9911-0563 